\title{
Optimal frequency window for Floquet engineering in optical lattices
}

\author{
Gaoyong Sun $\circledast^{1,2, *}$ and André Eckardt $\oplus^{1,3, \dagger}$ \\ ${ }^{1}$ Max-Planck-Institut für Physik komplexer Systeme, Nöthnitzer Straße 38, 01187 Dresden, Germany \\ ${ }^{2}$ College of Science, Nanjing University of Aeronautics and Astronautics, Nanjing 211106, China \\ ${ }^{3}$ Institut für Theoretische Physik, Technische Universität Berlin, Hardenbergstraße 36, 10623 Berlin, Germany
}

(Received 6 December 2019; accepted 28 January 2020; published 2 March 2020)

\begin{abstract}
The concept of Floquet engineering is to subject a quantum system to time-periodic driving in such a way that it acquires interesting properties. It has been employed, for instance, for the realization of artificial magnetic fluxes in optical lattices and, typically, it is based on two approximations. First, the driving frequency is assumed to be low enough to suppress resonant excitations to high-lying states above some energy gap separating a low-energy subspace from excited states. Second, the driving frequency is still assumed to be large compared to the energy scales of the low-energy subspace, so that also resonant excitations within this space are negligible. Eventually, however, deviations from both approximations will lead to unwanted heating on a time scale $\tau$. Using the example of a one-dimensional system of repulsively interacting bosons in a shaken optical lattice, we investigate the optimal frequency (window) that maximizes $\tau$. As a main result, we find that, when increasing the lattice depth, $\tau$ increases faster than the experimentally relevant timescale given by the tunneling time $\hbar / J$, so that Floquet heating becomes suppressed.
\end{abstract}

DOI: 10.1103/PhysRevResearch.2.013241

\section{INTRODUCTION}

The idea of Floquet engineering is to subject a quantum system to time-periodic driving in such a way that it acquires interesting properties that are difficult to achieve by other means. This concept has been applied very successfully to systems of atomic quantum gases in optical lattices [1]. The fact that these systems are extremely clean, well isolated from their environment, and highly tunable also in a timedependent fashion makes them an ideal platform for studying coherent many-body dynamics. Examples for Floquet engineering in optical lattices include, among others, dynamic localization [2,3], photon-assisted tunneling [4-8], the control of an interaction-induced quantum phase transition $[9,10]$, the creation of kinetic frustration [11,12], artificial magnetic fields [13-22], topological band structures [23-25], and number-dependent gauge potentials [26].

A simple explanation of the basic concept underlying Floquet engineering is often given by considering the one-cycle time-evolution operator

$$
\hat{U}(T, 0)=\mathcal{T} \exp \left[\frac{1}{i \hbar} \int_{0}^{T} d t \hat{H}(t)\right]
$$

*gysun@nuaa.edu.cn
$\dagger$ eckardt@pks.mpg.de

Published by the American Physical Society under the terms of the Creative Commons Attribution 4.0 International license. Further distribution of this work must maintain attribution to the author(s) and the published article's title, journal citation, and DOI. Open access publication funded by the Max Planck Society. for a quantum system described by a time-periodic Hamiltonian with angular driving frequency $\omega=2 \pi / T$,

$$
\hat{H}(t)=\hat{H}(t+T),
$$

where $\mathcal{T}$ denotes time ordering. The fact that this operator is unitary allows one, at least formally, to express it in terms of an Hermitian operator $\hat{H}_{F}$ that is called Floquet Hamiltonian,

$$
\hat{U}(T, 0) \equiv \exp \left(\frac{1}{i \hbar} T \hat{H}_{F}\right) .
$$

This effective time-independent Hamltonian $\hat{H}_{F}$ governs the time evolution of the system, when it is monitored stroboscopically in integer steps of the driving period $T$. Thus, at first glance, one might expect that the driven system behaves as some effective nondriven system described by the Hamiltonian $\hat{H}_{F}$. However, while the above reasoning applies to small quantum systems, the situation in many-body systems is more complex. Here the eigenstates of $\hat{H}_{F}$ will typically be superpositions of states having very different energies. This is a consequence of the lack of energy conservation in driven systems, which is reflected in the possibility of resonant coupling, and the fact that in a large system resonances will be ubiquitous. The lack of energy conservation suggests that in the thermodynamic limit the system approaches an infinitetemperature-like state, so that in the sense of eigenstate thermalization the eigenstates of $\hat{H}_{F}$ represent an infinitetemperature ensemble $[27,28]$. From this point of view, the Floquet Hamiltonian $\hat{H}_{F}$ does not seem to be a suitable object for engineering interesting system properties.

The fact that Floquet engineering can, nevertheless, be a useful concept also in many-body quantum systems, is related to the observation that in some parameter regimes the timescale $\tau$ associated with unwanted resonant processes, where the system absorbs (or emits) energy quanta $\hbar \omega$, 
can become rather long. Since typically energy absorption dominates, in the following, we will denote such detrimental energy-non-conserving processes as "heating" and $\tau$ as the corresponding "heating" time. ${ }^{1}$ On times shorter than $\tau$, we might be able to engineer and study interesting driving-induced physics described by an approximate timeindependent effective Hamiltonian $\hat{H}_{\text {eff }}$, corresponding to a nondriven system with modified properties. The standard strategy employed for deriving such an effective Hamiltonian involves two steps [9]:

The first step is given by a low-frequency approximation, where the assumption is made that the system remains in a low-energy subspace, which is separated by an energy gap from excited states, which is much larger than the driving frequency. In nondriven systems, such low-energy approximations are common. For example, in a lattice system, higherlying orbital states spanning Bloch bands above a band gap are neglected, when deriving Hubbard-type tight-binding models, or doublon-holon excitations lying above a charge gap of a Mott insulator are eliminated adiabatically, in order to derive spin Hamiltonians. For a sufficiently large energy gap, in nondriven systems one can expect that the admixture of higher lying states is captured by a converging perturbation theory and will always remain small. In contrast, in a periodically driven many-body system, the situation is generically different. Here resonant excitations to the neglected excited states can occur, where the drive provides one or several energy quanta $\hbar \omega$. Such processes contribute to the aforementioned detrimental heating. However, for driving frequencies (and amplitudes) much lower than the gap, so that the system would need to absorb many energy quanta $\hbar \omega$ ("photons") at once, they can be exponentially slow with respect to the photon number. Thus, by estimating the associated heating rate [29-38], we might be able to argue that we can still neglect higher lying states on the timescale of an experiment.

The second step is given by a high-frequency approximation. Let us assume that according to the first step we are able to neglect, say, higher lying Bloch bands, so that we can describe our system by a Hubbard Hamiltonian acting in the lowest Bloch band. Now, the periodic drive can still resonantly create excitations within this low-energy subspace. This form of energy absoprtion (heating) can be reduced considerably by considering driving frequencies that are sufficiently large, so that absorbing an energy quantum of $\hbar \omega$ corresponds to an exponentially slow high-order process in which several elementary excitations are created at once [39,40]. If this is the case, we can employ a rotating-wave approximate and describe the system by the time-averaged low-energy Hamiltonian (or compute also further corrections using a high-frequency expansion [40-44]). In this way, we arrive at an approximate effective Hamiltonian $\hat{H}_{\text {eff }}$ that describes the dynamics of our system on timescales before driving-induced heating sets in. The leading order of this expansion is simply given by the time-averaged Hamiltonian and corresponds to a rotating-wave approximation.

\footnotetext{
${ }^{1}$ This (rather common) terminology shall not imply that the system is described by thermodynamic variables such as temperature.
}

The two steps outlined above require that there is a window of suitable driving frequencies that are both low compared to the relevant energy gap separating the low-energy subspace from higher lying states and large compared to the energy scales governing this low-energy subspace. In this article, we investigate the question of whether such an optimal frequency window exists, using the experimentally relevant example of repulsively interacting bosonic atoms in a periodically shaken one-dimensional optical lattice. For this purpose, we compare the evolution generated by an approximate effective Hamiltonian $\hat{H}_{\text {eff }}$ acting in the lowest Bloch band to the evolution obtained from integrating the dynamics of the fully timedependent model that, apart from the lowest band, contains also first excited band.

The remaining part of this paper is organized as follows: After introducing the system and the model in Sec. II, in Sec. III we recapitulate the derivation of the approximate effective Hamiltonian $\hat{H}_{\text {eff }}$ from the low- and high-frequency approximations. In the following two sections, we then compare the evolution generated by $\hat{H}_{\text {eff }}$ to numerical simulations: In Sec. IV, we investigate the breakdown of the high-frequency approximation due to intraband heating and in Sec. V we study the combined effect of intraband and interband heating beyond the high- and low-frequency approximation. Finally, we close with Sec. VI.

\section{SYSTEM AND MODEL}

We consider a system of ultracold bosonic atoms in a onedimensional optical lattice potential

$$
V(\boldsymbol{r})=V_{0} \sin ^{2}\left(k_{L} x\right)+V_{\perp}(y, z) .
$$

Here, the laser wave number $k_{L}$ defines the recoil energy $E_{R}=$ $\hbar^{2} k_{L}^{2} /(2 m)$ with atom mass $m$, corresponding to the kinetic energy required to localize a particle on the length of a lattice constant $a=\pi / k_{L}$. Typical recoil energies take values of a few $\mathrm{kHz}$. The deep confining potential $V_{\perp}(y, z) \simeq \frac{m}{2} \omega_{\perp}^{2}\left(y^{2}+\right.$ $\left.z^{2}\right)$ shall reduce the dynamics to one spatial dimension via a large transverse excitation gap $\hbar \omega_{\perp}$ that freezes the particles in the lowest transverse single-particle state. More precisely, $\omega_{\perp}$ will be chosen large enough, so that the timescale for drivinginduced transverse heating can be expected to be much longer than the one for resonant excitations of longitudinal degrees of freedom in lattice direction, which we are going to investigate here.

The system shall be driven periodically in time by the homogenous sinusoidal force pointing in the lattice direction $\boldsymbol{e}_{x}$,

$$
\boldsymbol{F}(t)=-K a \cos (\omega t) \boldsymbol{e}_{x} .
$$

It is characterized by the driving strength $K$, corresponding to the amplitude of the potential offset between neighboring lattice sites, and the angular driving frequency $\omega$, which defines also the driving period $T=2 \pi / \omega$. Such a force can be realized as an inertial force by shaking the lattice back and forth in the $x$ direction.

In the absence of periodic forcing, experiments performed in the regime of deep lattices, $V_{0} / E_{R} \gtrsim 5$, at the typical ultracold quantum gas temperatures are described accurately 
by the single-band Bose Hubbard model [45]

$$
\hat{H}_{s}=-J_{s} \sum_{\ell=1}^{M-1}\left(\hat{b}_{s \ell+1}^{\dagger} \hat{b}_{s \ell}+\text { H.c. }\right)+\frac{U_{s}}{2} \sum_{\ell=1}^{M} \hat{n}_{s \ell}\left(\hat{n}_{s \ell}-1\right) .
$$

Here the index $\ell$ denotes the lattice sites in ascending order from 1 to $M$ and the label $s$ indicates the lowest Bloch band to be distinguished from the first excited band, labeled by $p$, which is considered below. Moreover, $\hat{b}_{\alpha \ell}^{\dagger}, \hat{b}_{\alpha \ell}$, and $\hat{n}_{\alpha \ell}=\hat{b}_{\alpha \ell}^{\dagger} \hat{b}_{\alpha \ell}$ denote the creation, annihilation, and number operator for a boson in a Wannier state of band $\alpha$ on site $\ell$. Nearest-neighbor tunneling is described by the parameter $J_{s}$ and on-site interactions by the Hubbard parameter $U_{s}$.

While in a nondriven system, a description in the lowenergy subspace of the $s$ band is well justified, this assumption is not as clear in a system that is driven periodically. Even if the driving frequency is small compared to the band gap separating the $s$ band from the first excited $p$ band, states of excited bands might still be populated via multiphoton excitations corresponding to either single-particle processes [30,35] or two-particle scattering [36]. If periodic driving is used to control the physics of the lowest band, such excitation processes must be viewed as unwanted heating. In order to estimate this effect, we will also take into account the first excited band, which for the undriven lattice is captured by the Hamiltonian

$$
\begin{aligned}
\hat{H}_{p}= & \Delta \sum_{\ell=1}^{M} \hat{n}_{p \ell}+J_{p} \sum_{\ell=1}^{M-1}\left(\hat{b}_{p \ell+1}^{\dagger} \hat{b}_{p \ell}+\text { H.c. }\right) \\
& +\frac{U_{p}}{2} \sum_{\ell=1}^{M} \hat{n}_{p \ell}\left(\hat{n}_{p \ell}-1\right),
\end{aligned}
$$

and coupled to the $s$ band via the interband interaction term

$$
\hat{H}_{\mathrm{sp}}=U_{\mathrm{sp}} \sum_{\ell=1}^{M}\left[2 \hat{n}_{s \ell} \hat{n}_{p \ell}+\frac{1}{2}\left(\hat{b}_{p \ell}^{\dagger} \hat{b}_{p \ell}^{\dagger} \hat{b}_{s \ell} \hat{b}_{s \ell}+\text { H.c. }\right)\right] \text {. }
$$

Here $\Delta$ denotes the orbital energy required to excite a particle to a Wannier state of the $p$ band and $J_{p}$ and $U_{p}$ describe nearest-neighbor tunneling and on-site interactions in this $p$ band, respectively. The on-site scattering and repulsion between $s$ and $p$ states is quantified by $U_{\mathrm{sp}}$.

If the energy scales of the periodic force, $\hbar \omega$ and $K$, remain below the band gap $\Delta$, the bands of the undriven problem, $s$ and $p$, provide a useful basis also for the description of the driven system (see the supplemental material of Ref. [36]). Assuming this regime, we project the potential $-\boldsymbol{r} \cdot \boldsymbol{F}(t)$ induced by the force to the lowest two bands and obtain the driving term of the Hamiltonian:

$$
\hat{H}_{\mathrm{dr}}(t)=K \cos (\omega t) \sum_{\ell=1}^{M}\left[\ell\left(\hat{n}_{s \ell}+\hat{n}_{p \ell}\right)+\eta\left(\hat{b}_{p \ell}^{\dagger} \hat{b}_{s \ell}+\text { H.c. }\right)\right]
$$

where $\eta$ is the dipole matrix element between two Wannier states of the $s$ and the $p$ band on the same lattice site in units of the lattice constant.

The total Hamiltonian to be used for our analysis is now given by

$$
\hat{H}(t)=\hat{H}_{s}+\hat{H}_{p}+\hat{H}_{\mathrm{sp}}+\hat{H}_{\mathrm{dr}}(t) .
$$

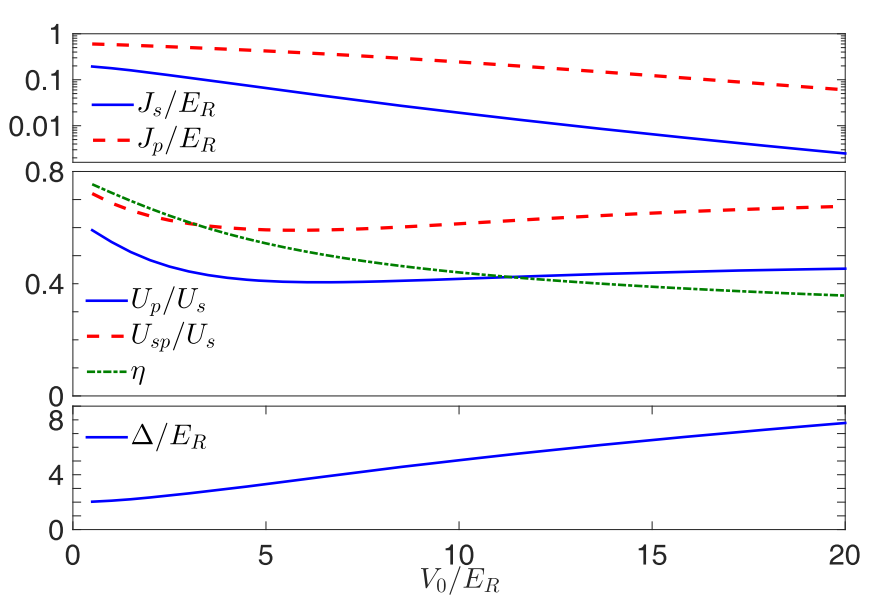

FIG. 1. Parameters characterizing the two-band Bose-Hubbard model for a shaken one-dimensional optical cosine lattice plotted vs the lattice depth $V_{0} / E_{R}$.

The number of independent parameters that describe this model is reduced considerably by noticing that $J_{s} / E_{R}, J_{p} / E_{R}$, $\Delta / E_{R}$, and $\eta$ are determined completely by the dimensionless lattice depth $V_{0} / E_{R}$. Moreover, the interaction parameters $U_{s}$, $U_{p}$, and $U_{\text {sp }}$ share the very same (linear) dependence on both the $s$-wave scattering length $a_{s}$ (which can be tuned using Feshbach resonances) and the transverse confinement $\omega_{\perp}$, so that their ratios $U_{p} / U_{s}$ and $U_{\mathrm{sp}} / U_{s}$ equally depend on $V_{0} / E_{R}$ only. Thus, taking $J_{s}$ and $\hbar / J_{s}$ as the units for energy and time, respectively, the undriven model is characterized by $V_{0} / E_{R}$ and $U_{s} / J_{s}$ as well as by the average number of particles per site $N / M$. The periodic driving is furthermore characterized by the dimensionless diving strength $K / J_{S}$ and angular frequency $\hbar \omega / J_{s}$. The dependence of the model parameters on the lattice depth $V_{0} / E_{R}$, obtained from band-structure calculations, is shown in Fig. 1.

\section{LOW- AND HIGH-FREQUENCY APPROXIMATIONS}

Most schemes of Floquet engineering in optical lattices (such as, for example, the control of the bosonic Mott transition $[9,10]$, the implementation of kinetic frustration $[11,12]$, the creation of artificial gauge fields [13,15-19,22], and the realization of Floquet topological insulators [23-25]) are based on two approximations: a low-frequency approximation with respect to orbital degrees of freedom and a high-frequency approximation with respect to processes occurring in the lowest band described by $H_{s}$.

The low-frequency single-band approximation is based on the assumption that the driving frequency and amplitude remain low enough to ensure that the system remains in the subspace spanned by the lowest ( $s$-type) Wannier-like orbital at each lattice site. It roughly requires driving frequencies

$$
\hbar \omega \ll \Delta
$$

and driving amplitudes $K$ smaller than a threshold value $K_{\text {th }}$ below which multiphoton transitions are expected to be suppressed exponentially with the photon number $\Delta / \hbar \omega$ [35]. It leads to a description of the system in terms of a tight-binding model with a single orbital state per lattice site, which in our 
case is given by the single-band model

$$
\hat{H}_{\mathrm{sb}}(t)=\hat{H}_{s}+K \cos (\omega t) \sum_{\ell=1}^{M} \ell \hat{n}_{s \ell} .
$$

The high-frequency approximation is based on the assumption that the driving frequency is still large compared to the energy scales $J_{s}$ and $U_{s}$ governing the low-energy model (12),

$$
\hbar \omega \gg J_{s}, U_{s} .
$$

Under these conditions, the resonant creation of collective excitations of energy $\hbar \omega$ becomes a slow high-order process that can be neglected on sufficiently short timescales. This allows us to describe the system using an approximate effective time-independent Hamiltonian obtained from a highfrequency expansion $[9,40,43,44]$. For that purpose, we first perform a gauge transformation with the time-periodic unitary operator

$$
\hat{U}(t)=\exp \left(-i \sum_{\ell=1}^{M} \theta(t) \ell \hat{n}_{s \ell}\right)
$$

with $\theta(t)=K /(\hbar \omega) \sin (\omega t) \ell$, which integrates out the driving term. The transformed Hamiltonian $\hat{H}^{\prime}=\hat{U}^{\dagger} \hat{H}_{\mathrm{sb}} \hat{U}-i \hat{U}^{\dagger} \dot{\hat{U}}$ reads

$\hat{H}^{\prime}(t)=-J_{s} \sum_{\ell=1}^{M-1}\left(e^{i \theta(t)} \hat{b}_{s \ell+1}^{\dagger} \hat{b}_{s \ell}+\right.$ H.c. $)+\frac{U_{s}}{2} \sum_{\ell=1}^{M} \hat{n}_{s \ell}\left(\hat{n}_{s \ell}-1\right)$.

The fact that it possesses typical matrix elements that are small compared to $\hbar \omega$ even for large $K \sim \hbar \omega$ justifies the high-frequency approximation also for strong driving. Its leading order is given by the rotating-wave approximation, where the system is described by the time-averaged Hamiltonian

$$
\begin{aligned}
\hat{H}_{\mathrm{eff}} & =\frac{1}{T} \int_{0}^{T} d t \hat{H}^{\prime}(t) \\
& \left.=-J_{s}^{\mathrm{e} f f} \sum_{\ell=1}^{M-1}\left(\hat{b}_{s \ell+1}^{\dagger} \hat{b}_{s \ell}+\text { H.c. }\right)+\frac{U_{s}}{2} \sum_{\ell=1}^{M} \hat{n}_{s \ell}\left(\hat{n}_{s \ell}-1\right)\right] .
\end{aligned}
$$

Here the effective tunneling matrix element

$$
J_{s}^{\text {eff }}=J_{s} \mathcal{J}_{0}(K / \hbar \omega)
$$

acquired a dependence on the scaled driving amplitude $K /(\hbar \omega)$ described by a Bessel function $\mathcal{J}_{n}$. In this way, the time evolution of the system's state $|\psi(t)\rangle$ is approximately described by

$$
|\psi(t)\rangle \approx \hat{U}(t) e^{-\frac{i}{\hbar}\left(t-t_{0}\right) \hat{H}_{\mathrm{eff}}} \hat{U}^{\dagger}\left(t_{0}\right)\left|\psi\left(t_{0}\right)\right\rangle .
$$

In particular, we expect

$$
|\psi(n T)\rangle \approx e^{-\frac{i}{\hbar} n T \hat{H}_{\mathrm{eff}}}|\psi(0)\rangle \equiv\left|\psi_{n}^{\mathrm{eff}}\right\rangle
$$

for integers $n$, when monitoring the dynamics stroboscopically in steps of the driving period at those times $t=n T$, for which $\hat{U}(n T)=1$. Higher orders of the high-frequency expansion will provide relative corrections of the order of $J_{s} / \hbar \omega$ to the evolution governed by $\hat{H}_{\text {eff }}[40,43]$.
The single-band high-frequency approximation, leading to a description of the system's dynamics in terms of the approximate effective Hamiltonian (16), requires that there is a window of driving frequencies for which both conditions (11) and (13) are fulfilled. Since with increasing lattice depth $V_{0} / E_{R}$ both $J_{s}$ decreases rapidly and $\Delta$ increases moderately (see Fig. 1), while the interaction parameter $U_{s}$ can be made small by tuning the $s$-wave scattering length using a Feshback resonance, such a window will open for sufficiently large $V_{0} / E_{R}$. However, even within such a frequency window, heating will not be suppressed completely and will eventually make itself felt on some timescale $\tau$. This heating time $\tau$ has to be compared to the typical duration of an experiment, which will be given by some fixed multiple of the tunneling time $\hbar / J_{s}$, which in turn increases exponentially with the lattice depth (asymptotically for deep lattices $\ln \left(J_{s} / E_{\mathrm{R}}\right) \simeq-2 \sqrt{V_{0} / E_{\mathrm{R}}}$ [46]; see also Fig. 1). Thus, in order to take into account also this latter effect, in the following we will investigate the behavior of the dimensionless heating time $\tau J_{s} / \hbar$. In doing so, we have to keep in mind that there will also be background heating (resulting from noise, three-body collisions, or scattering with background particles), which is independent of the periodic driving and happens on some timescale $\tau_{0}$. Assuming $\tau_{0} \sim 1 \mathrm{~s}(\sim 10 \mathrm{~s})$, requiring $\tau_{0} \gg \hbar / J_{s}$, and noting that $E_{R} \sim 2 \pi \hbar 3 \mathrm{kHz}$ for typical experiments, we can see from Fig. 1 that the lattice depth is limited to values $V_{0} / E_{R} \lesssim 15(20)$.

\section{INTRABAND HEATING}

Let us first investigate the validity of the high-frequency approximation, before considering also heating due to the coupling to the first excited band. For this purpose, we consider the following quench scenario. We assume that the system is prepared in the ground state of the undriven Hamiltonian (6), when at time $t=0$ the driving amplitude is switched on abruptly to a finite value $K$. We integrate the time evolution of the system described by the time-dependent single-band Hamiltonian $\hat{H}_{\mathrm{sb}}(t)$ [Eq. (12)] and compare it to the approximate solution $\left|\psi_{n}^{\text {eff }}\right\rangle$ [Eq. (19)] obtained from the timeaveraged single-band Hamiltonian $\hat{H}_{\text {eff }}$. For that purpose, we consider a small system of $N=6$ particles on $M=10$ lattice sites, for which we can integrate the time evolution exactly.

In order to monitor the deviation between the exact time evolution and the dynamics predicted by the rotating-wave approximation, we consider the expectation value

$$
n_{0}(t)=\left\langle\hat{a}_{s 0}^{\dagger} \hat{a}_{s 0}\right\rangle \quad \text { with } \quad \hat{a}_{s 0}=\frac{1}{\sqrt{M}} \sum_{\ell=1}^{M} \hat{b}_{s \ell},
$$

which corresponds to the mean occupation of the singleparticle state with quasimomentum 0 in the $s$ band. The difference

$$
\Delta n_{0}(t)=n_{0}(t)-n_{0}^{\mathrm{eff}}(t)
$$

between the exact expectation value and the one obtained within the rotating-wave approximation taken at times $t=n T$ with integer $n$ will serve as an indicator for the validity of the approximations made. While for the results presented in this section, $n_{0}(t)$ refers to the dynamics generated by the 


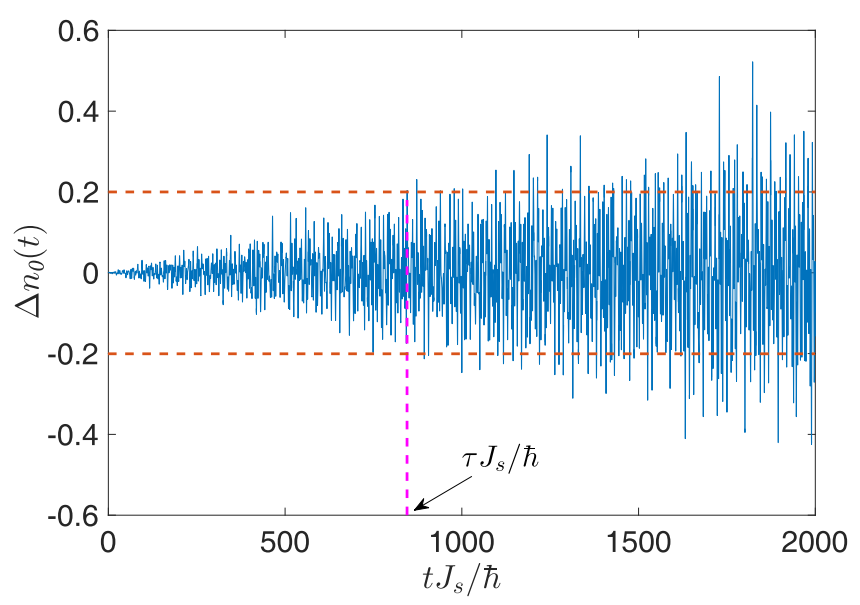

FIG. 2. Difference $\Delta n_{0}$ between the exact time evolution of the single-band Hamiltonian (12) and that obtained from the rotatingwave approximation (19), taken at times $t=n T$ with integer $n$. The time evolution is initiated by abruptly switching the amplitude of the drive at $t=0$ from 0 to $K$. The parameters are $N=6, M=10$, $V_{0} / E_{R}=14, U / J_{s}=1, \hbar \omega / J_{s}=30$, and $K / \hbar \omega=4$. At time $\tau$, the difference $\Delta n_{0}$ exceeds 0.2 for the first time.

time-dependent single-band Hamiltonian (12), later on in the following section, $n_{0}(t)$ will correspond to the dynamics of the full driven two-band model (10).

In Fig. 2 we plot $\Delta n_{0}(t)$ for a quench to a large driving amplitude $K / \hbar \omega=4$ (the other parameters are specified in the caption). For this value, the effective tunneling parameter changes its sign, $J_{s}^{\text {eff }} \approx-0.4 J$, so that the quench is significant also on the level of the rotating-wave approximation. We can see that $\Delta n_{0}(t)$ shows an irregular oscillatory behavior, with a roughly linearly growing envelope. We define the heating time $\tau$ as the time at which $\left|\Delta n_{0}(t)\right|$ exceeds the value $\Delta n_{\text {cut }}=0.2$ for the first time. Note that $\tau$ gives only an estimate for the timescale on which heating starts to play a role. The value of $\Delta n_{\text {cut }}$ is obviously somewhat arbitrary. It is chosen to be much smaller than the initial occupation of the zero momentum state, which is of the order of $N$, and it is also smaller than (and of the order of) the filling factor $N / M=0.6$ corresponding to the mean occupation of each momentum state. The linear spreading of the envelope of $\Delta n_{0}(t)$ implies that altering $\Delta n_{\text {cut }}$ by a factor of order 1 will simply alter the heating time $\tau$ by roughly the same factor. Note also that the typical deviations $\left|\Delta n_{0}(t)\right|$ at time $t=\tau$ are smaller than $\Delta n_{\text {cut }}=0.2$, since in most cases $\Delta n_{\text {cut }}$ is reached the first time during the time evolution when an extreme fluctuation of $\left|\Delta n_{0}(t)\right|$ occurs.

Note that, alternatively, the time $\tau$ could also be defined via the (stroboscopic or period-averaged) energy absorption. Such a definition would possess the advantage that, to some extent, in experiments it can be measured (or at least estimated) directly from time-of-flight images [30,36-38]. On the other hand, from the point of view of Floquet engineering, the relevant quantity to look at is the deviation from the approximate effective Hamiltonian, the physics of which we wish to implement. These deviations are not necessarily proportional to the absorbed energy. Namely, the excitation of a particle within the lowest band might be as detrimental as its excitation

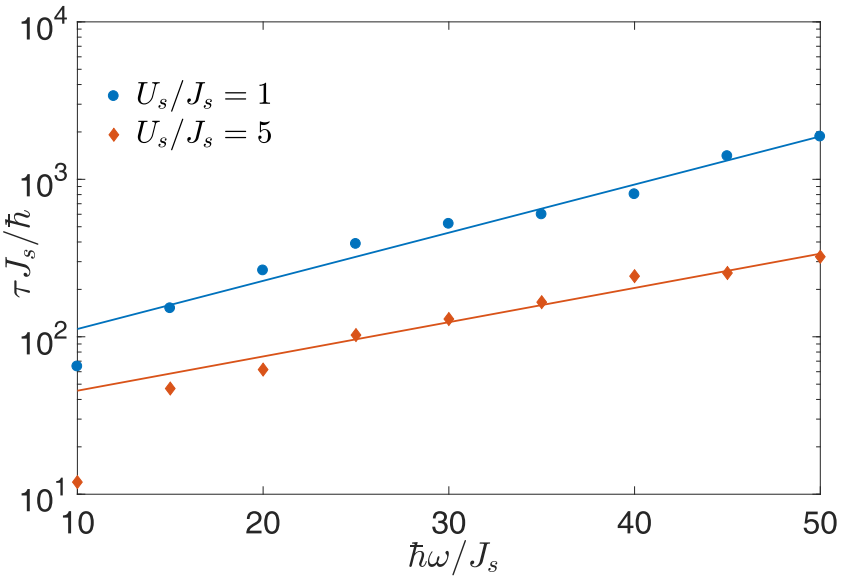

FIG. 3. Heating time $\tau$ (dots) vs driving frequency $\hbar \omega / J_{s}$ for two different values of the interaction strength $U_{s} / J_{s}$. The other parameters are chose as in Fig. 2: $N=6, M=10, K / \hbar \omega=4$, and $V_{R} / E_{R}=14$. The solid lines are exponential fits.

to the first excited band via a multiphoton process, despite the fact that the former is associated with a much lower energy absorption than the latter. Therefore, we have decided to define the "heating" time $\tau$ via deviations from the dynamics expected from the target Hamiltonian, as described in the previous paragraph.

In Fig. 3, we plot the heating time $\tau J_{s} / \hbar$ versus the driving frequency $\hbar \omega / J$ for two different values of the interaction strength $U / J_{s}=1$ and $U / J_{s}=5$ (the other parameters are specified in the caption). We see that the heating time is considerably reduced for the larger value of the interactions. Moreover, an exponential dependence of the heating time on the driving frequency can be observed. This agrees with the expectation for heating processes based on perturbation theory in Floquet space [39]. Namely, one can argue that the order of the process of absorbing an energy quantum $\hbar \omega$, corresponding to the number of elementary excitations (quasiparticles) that have to be collectively excited, will grow like a power of $\omega$ and that the corresponding matrix element will be suppressed exponentially with the order $[39,40]$. Such an exponential suppression of heating with respect to the driving frequency has recently also been proven for spin systems having a finite local energy bound [47,48]. Note, however, these proofs do not apply to the bosonic Hubbard model considered here, which in principle allows for macroscopic site occupations.

\section{INTRABAND AND INTERBAND HEATING}

The exponential increase of the heating time with respect to the driving frequency visible in Fig. 3 is an artifact of the single-band description of the driven lattice system. Namely, for sufficiently large driving frequencies unwanted excitations to higher lying orbital states (spanning excited Bloch bands) will become the dominant heating effect. In order to take into account this effect, we will include also the coupling to the $p$ band. For this purpose, we consider the two-band Hamiltonian (10) and monitor the heating time $\tau$ defined in the same way as in the previous section. In 


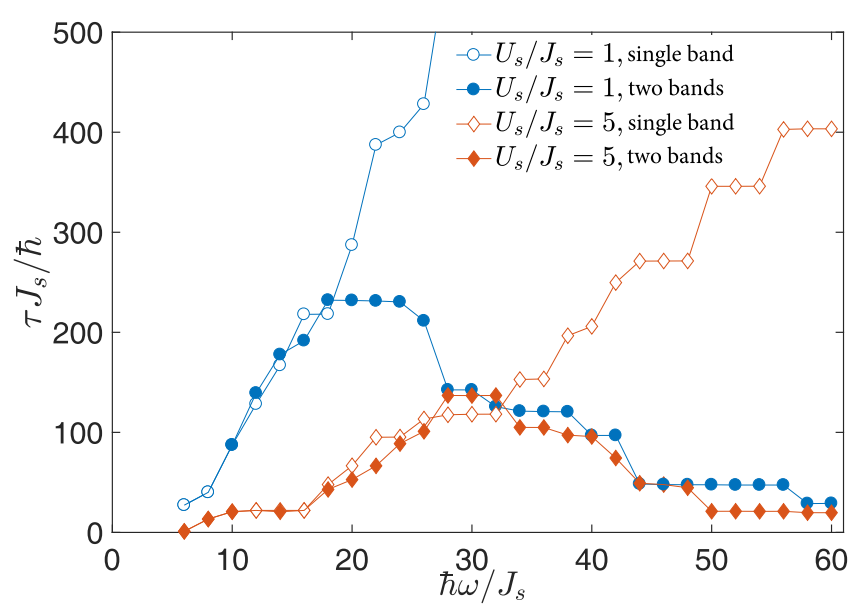

FIG. 4. Heating time vs driving frequency both for the singleband Hamiltonian (empty symbols) and the two-band Hamiltonian (full symbols) for a system of $N=4$ particles on $M=8$ sites (corresponding to 16 single-particle states) with $V_{0} / E_{R}=14, K / \hbar \omega=4$, and two different interaction strengths.

an experiment, of course, also further (higher lying) bands will play a role. However, the coupling to the first excited band is most dominant, because with respect to the lowest band it both is energetically closest and possesses the largest coupling matrix elements. Therefore, the characteristic timescale for the interband heating processes is determined by transitions to the $p$ band. Higher lying bands can still make themselves felt, e.g., in the precise shape of resonance lines (as discussed in Ref. [30]). However, such details are not crucial for the present analysis, which is interested in the timescales only.

In Fig. 4, we plot the heating time $\tau$ versus the driving frequency for a system of $N=4$ particles on $M=8$ sites (corresponding to 16 single-particle states) with lattice depth $V_{0} / J=14$. For strong driving, $K / \hbar \omega=4$, and two different interaction strengths, $U_{s} / J_{s}=1$ and $U_{s} / J_{s}=5$, we compare the heating times obtained from the single-band model (12) (open circles) to those obtained from the two-band model (10) (filled circles). As expected, we can observe that, while the coupling to the $p$ band does not influence the heating time for low frequencies, it becomes dominant for large driving frequencies. For the two-band model, the interplay between intraband and interband heating gives rise to a maximum of the heating time, $\tau_{\mathrm{opt}}$, at some optimal intermediate driving frequency $\omega_{\mathrm{opt}}$. For the larger interaction strength, $\tau_{\mathrm{opt}}$ is lower and occurs at a larger frequency.

To study the impact of interactions in more detail, we compare the frequency-dependent heating times for various interaction strengths $U_{s} / J_{s}$ in Fig. 5. The inset shows the optimal (maximum) heating time $\tau_{\mathrm{opt}}$ (diamonds, right axis) and the corresponding optimal driving frequency $\omega_{\text {opt }}$ (circles, left axis) versus $U_{s} / J_{s}$. We observe a significant reduction of $\tau_{\text {opt }}$ combined with an upshift of $\omega_{\text {opt }}$, when increasing the interaction strength $U_{s} / J_{s}$ up to values of about 3. Both the shift of $\omega_{\text {opt }}$ and the noticeable reduction of $\tau_{\text {opt }}$ for the singleband model (Fig. 3) suggest that increasing the interactions mainly enhances intraband heating, so that intraband heating becomes the dominant heating processes limiting $\tau$ up to

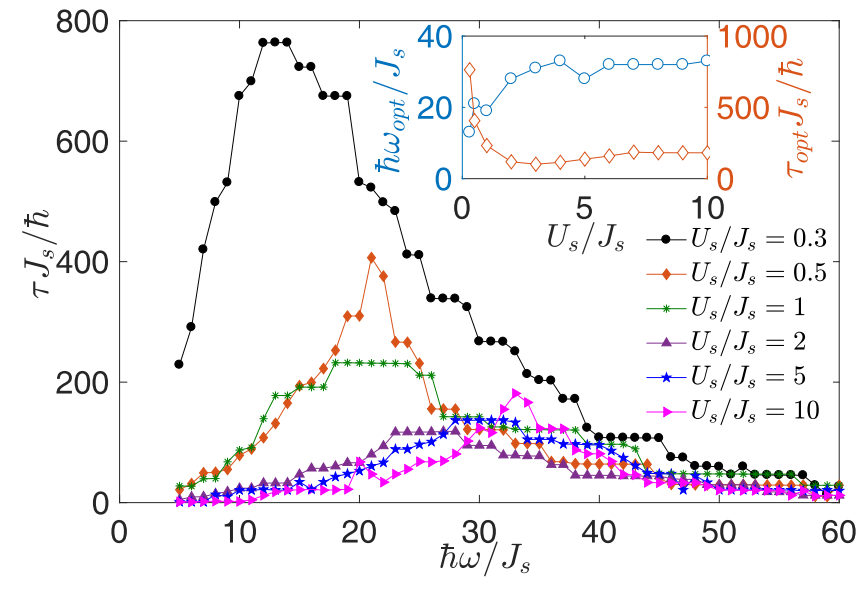

FIG. 5. Heating time $\tau J_{s} / \hbar$ vs driving frequency $\hbar \omega / J_{s}$ for the two-band model with different interaction parameters $U_{s} / J_{s}$, for $N=$ $4, M=8, V_{0} / E_{R}=14$, and $K / \hbar \omega=4$. The inset shows the optimal (maximum) heating time $\tau_{\mathrm{opt}}$ (diamonds) and the corresponding optimal frequency $\omega_{\text {opt }}$ vs $U_{s} / J$.

larger values of $\omega$. For values of $U_{s} / J_{s}$ that are larger than 3 , both $\tau_{\mathrm{opt}}$ and $\omega_{\mathrm{opt}}$ approximately saturate. We attribute this favorable behavior to the reaching of the strongly interacting regime $U_{s} /\left|J_{s}^{\text {eff }}\right| \approx 2.5\left(U_{s} / J_{s}\right) \gg 1$ in the lowest band. Here the kinetic energy of the particles is not sufficient anymore to induce changes in the site occupations that are associated with a change of interaction energy (for an initial state without multiply occupied sites this regime corresponds to the hardcore boson limit). Once this regime is reached, the physics within the lowest band does not change much anymore, when the interactions are increased further, which is consistent with the observed saturation. This argument holds until eventually for even stronger interactions, $U_{\mathrm{sp}} \sim \Delta$, deviations due to interband coupling will make themselves felt.

In Fig. 6, we depict the lowest-band zero-quasimomentum occupation $n_{0}(t)$ in units of its initial value $n_{0}(0)$ at time $t \approx 40 \hbar / J_{s}$. This time is chosen to be large compared to the tunneling time $\hbar / J_{s}$, which is the relevant timescale for experiments. It is plotted versus the interaction strength $U_{s} / J_{s}$ and the driving frequency $\hbar \omega / J_{s}$, where the low-frequency regime is shown in the left panel, while results for higher driving frequencies are given in the right panel. In the underlying simulations, we have considered a lattice depth of $V_{0} / E_{\mathrm{R}}=10$ and a driving strength of $K / \hbar \omega=1.5$, which is smaller than the one used previously and does not induce a sign change of the effective tunneling matrix element $(17), J_{s}^{\text {eff }} \approx 0.51 J_{s}$. The latter implies that on the level of the effective Hamiltonian, the quench induced when switching on the driving does not correspond to an inversion of the effective dispersion relation but rather to a reduction of the band width by a factor of one half. On the level of the time-averaged Hamiltonian (16), this rather mild quench will excite the system only weakly, so that the occupation $n_{0}(t) / n_{0}(0)$ will retain a rather large value also during the dynamics following the quench. Thus, a significant reduction of $n_{0}(t) / n_{0}(0)$ indicates unwanted driving-induced heating. Note also that (for fixed $K / \hbar \omega$ ) the ideal dynamics generated by $\hat{H}_{\text {eff }}$, and thus also $n_{0}(t) / n_{0}(0)$, should be independent of the driving frequency. Therefore, 


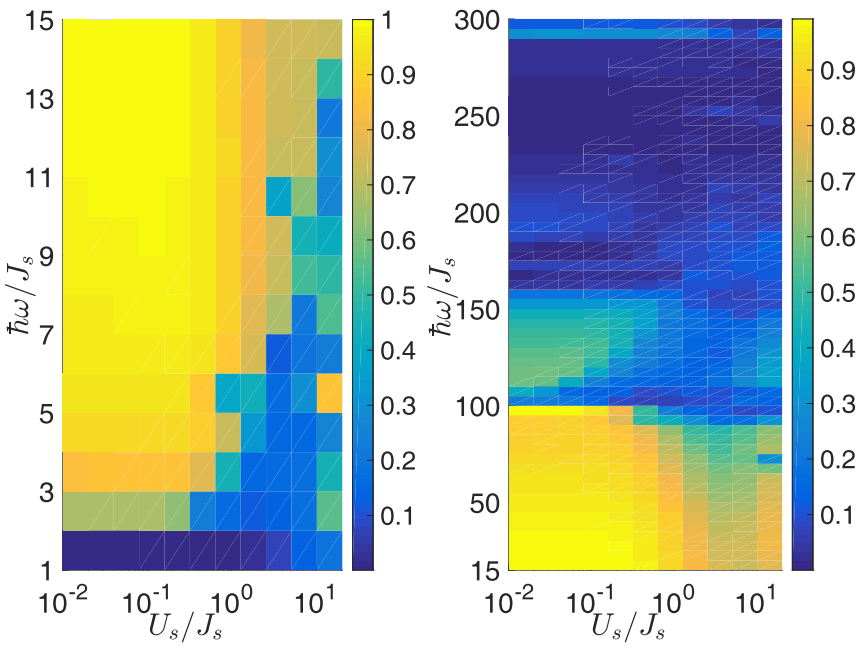

FIG. 6. Map of $n_{0}(t) / n_{0}(0)$ at time $t=100 \mathrm{~ms}$ for the twoband model vs driving frequency and interaction strengths, with $N=4, M=8, V_{0} / E_{R}=10$, and $K / \hbar \omega=1.5$. Here $n_{0}(t)$ is defined in Eq. (20). We assumed a recoil energy of $E_{R}=3.332 \pi \hbar \mathrm{kHz}$, a typical value for an experiment with ${ }^{87} \mathrm{Rb}$ atoms, for which the chose time span corresponds to $t J_{s} / \hbar \approx 40$ tunneling times. The driving strength corresponds to an effective tunneling matrix element of $J_{s}^{\text {eff }} \approx 0.5 J_{s}$.

also any frequency dependence of $n_{0}(t) / n_{0}(0)$ must be viewed as a deviation from the target dynamics generated by $\hat{H}_{\text {eff }}$.

In Fig. 6, we find signatures of heating in the form of a significant reduction of $n_{0}(t) / n_{0}(0)$ in various regimes. In the regime of weak interactions $U_{s} / J_{s} \ll 1$, heating is visible both for too low frequencies, when $\hbar \omega \sim J_{s}$, as well as for too high frequencies, when $\hbar \omega \sim \Delta$ (with $\Delta / J_{s} \sim 250$ for the given lattice depth). When the interband interactions $U_{s}$ become larger than the interband tunneling $J_{s}$, low-frequency heating sets in already for larger $\hbar \omega$, in accordance with condition (11). At the same time, we can also observe that interband heating at large frequencies is enhanced in the presence of interactions. For the chosen lattice depth of $V_{0} / E_{\mathrm{R}}=10$, we observe that strong interactions $U_{s} / J_{S} \gg 1$ lead to significant heating at any frequency.

The dependence of the heating time $\tau J_{S} / \hbar$ on the lattice depth $V_{0} / E_{\mathrm{R}}$ is investigated in detail in Fig. 7, where we plot the scaled heating time $\tau J_{s} / \hbar$ versus $\hbar \omega / J_{s}$ for various values of $V_{0} / J_{s}$ and for $U_{s} / J_{s}=5$ as well as $K / \hbar \omega=4$. The inset shows $\tau_{\mathrm{opt}} J_{s} / \hbar$ and $\hbar \omega_{\mathrm{opt}} / J_{s}$ versus $V_{0} / E_{\mathrm{R}}$. We can observe that both $\tau_{\text {opt }} J_{s} / \hbar$ and $\hbar \omega_{\text {opt }} / J_{s}$ increase with the lattice depth. The main figure shows that this behavior is associated with a significant reduction of heating for large $\hbar \omega / J_{s}$. Let us discuss this behavior in more detail.

First, we can notice that the intraband dynamics, described by the single-band Hamiltonian (12) and measured in the natural unit of the tunneling time $\hbar / J_{s}$, is determined by the dimensionless ratios $U_{s} / J_{s}, \hbar \omega / J_{s}$, and $K / \hbar \omega$, which we kept fixed in our simulations when increasing the lattice depth $V_{0} / E_{\mathrm{R}}$. This choice of fixed parameters is natural from the point of view of quantum simulation, where we wish to engineer the properties of the lowest band described by the approximate effective Hamiltonian (16). It explains why for small $\hbar \omega / J_{s}$, for which interband coupling is negligible, the

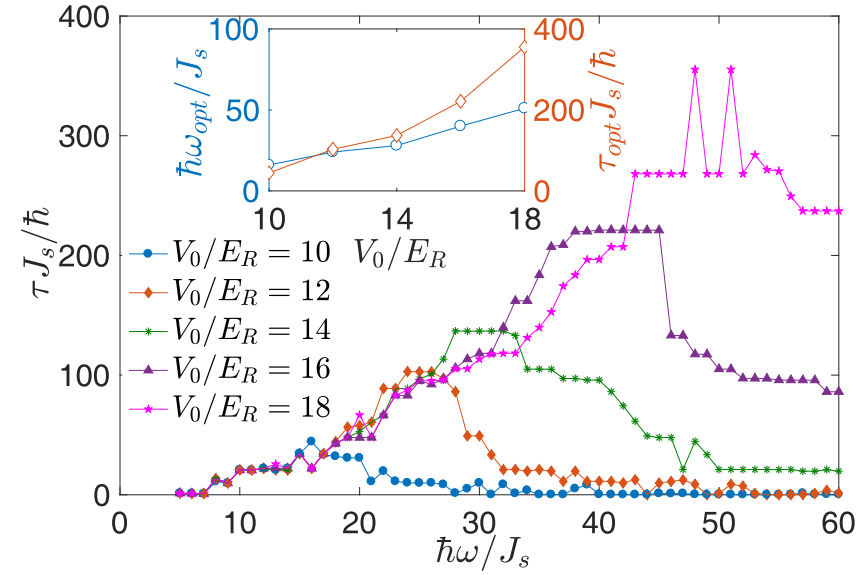

FIG. 7. Heating time $\tau J_{s} / \hbar$ vs driving frequency $\hbar \omega / J_{s}$ for the two-band model with different lattice depths $V_{0} / E_{R}$, for $N=4$, $M=8, U_{s} / J_{s}=5$, and $K / \hbar \omega=4$. The inset shows the optimal (maximum) heating time $\tau_{\mathrm{opt}}$ (diamonds) and the corresponding optimal frequency $\omega_{\mathrm{opt}} \mathrm{vs} V_{0} / E_{R}$.

dimensionless heating time $\tau J_{s} / \hbar$ is hardly influenced by the lattice depth. This can be seen from the fact that all curves in Fig. 7 agree up to the point $\left(\sim \hbar \omega_{\text {opt }} / J_{S}\right)$, where $\tau J_{s} / \hbar$ starts to be reduced by interband processes.

We can, moreover, observe in Fig. 7 that the interband heating, which is responsible for the reduction of $\tau J_{s} / \hbar$ at large frequencies, is significantly reduced with increasing lattice depth. This behavior results from the interplay of various effects. On the one hand, with increasing lattice depth $V_{0} / E_{\mathrm{R}}$ the band separation $\Delta / E_{\mathrm{R}}$ increases, whereas the interband coupling parameter $\eta$ decreases (Fig. 1). Both effects tend to reduce interband heating. An additional and much stronger reduction of interband heating, however, results from the exponential suppression of the tunneling parameter $J_{s}$ with the square root of the lattice depth $V_{0} / E_{\mathrm{R}}$ (Fig. 1). Namely, since we keep the dimensionless ratio $\hbar \omega / J_{S}$ fixed (taking the point of view of quantum simulation, as explained in the previous paragraph), the number $n_{\mathrm{ph}}$ of photons (i.e., energy quanta $\hbar \omega$ ) needed to overcome the band separation $\Delta, n_{\mathrm{ph}} \approx \Delta /(\hbar \omega)$, will strongly increase with the lattice depth. This, in turn, implies a very strong suppression of interband heating, since we expect an exponential suppression of interband transitions with $n_{\mathrm{ph}}[30,35]$.

The effects described in the previous paragraph explain a strong increase of the heating time $\tau$ with increasing lattice depth. However, from the point of view of quantum simulation, we have to compare the heating time to the relevant experimental timescale, given by the tunneling time $\hbar / J_{s}$. This is why here we are always plotting the scaled heating time $\tau J_{s} / \hbar$. Therefore, when increasing the lattice depth, the expected strong increase of $\tau$ directly competes with the exponential increase of $\hbar / J_{s}$ with the square root of the lattice depth. The results presented in Fig. 7 clearly show that the former effect wins over the latter one, so that, all in all, $\tau J_{S} / \hbar$ is reduced when the lattice depth $V_{0} / E_{\mathrm{R}}$ is raised. We can, thus, see a noticeable increase of the optimal heating time $\tau_{\text {opt }} J_{s} / \hbar$ (shown in the inset of Fig. 7) with $V_{0} / E_{R}$.

While the results of Fig. 7 imply that driving-induced heating can effectively be reduced by raising the lattice depth, 


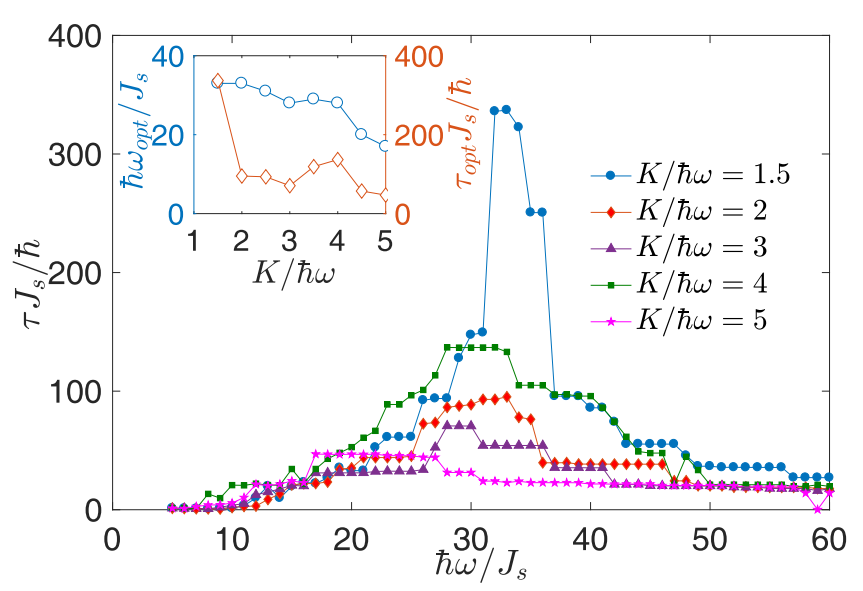

FIG. 8. Heating time $\tau J_{s} / \hbar$ vs driving frequency $\hbar \omega / J_{s}$ for the two-band model with different driving amplitudes $K / \hbar \omega$, for $N=4$, $M=8, V_{0} / E_{R}=14$, and $U_{s} / J_{s}=5$. The inset shows the optimal (maximum) heating time $\tau_{\mathrm{opt}}$ (diamonds) and the corresponding optimal frequency $\omega_{\text {opt }}$ vs $K / \hbar \omega$.

this possibility is limited by non-driving-induced heating processes, originating, e.g., from three-body collisions, scattering with background particles, or noise. Namely, the tunneling time, which increases with the lattice depth, has to remain short compared to the timescale $\tau_{0}$ associated with such background heating. In turn, this means that by increasing $\tau_{0}$ by reducing non-driving-induced heating, the experimentalist can also reduce driving-induced heating. This is a major result of this article.

Let us, finally, also have a look at the dependence of the heating time on the driving strength. In Fig. 8, we plot $\tau J_{s} / \hbar$ versus $K / \hbar \omega$ for a system with $V_{0} / E_{\mathrm{R}}=14$ and $U_{s} / J_{s}=$ 5. We focus on values of $K / \hbar \omega$ that are interesting for Floquet engineering (i.e., that are large enough to achieve a significant modification of $J_{s}^{\text {eff }}$ and not much larger than required for tuning $J_{s}^{\text {eff }}$ to negative values). For the smallest considered driving strength of $K / \hbar \omega=1$, a narrow window of frequencies is found for which the heating time takes large values of more than 300 tunneling times. This window disappears for stronger driving. Note that we do not find a simple monotonous decrease of the heating time with respect to the driving strength. We attribute this observation to the nonmonotonous behavior of the finite-frequency components $\propto e^{i m \omega t}$ of the time-dependent Hamiltonian (15) in the rotating frame (as well as of the corresponding two-band Hamiltonian). Namely, these terms, which describe heating processes beyond the rotating wave approximation (16) where the system exchanges $m$ energy quanta $\hbar \omega$, involve Bessel-function expressions $\mathcal{J}_{m}(K / \hbar \omega)$ that depend in a nonmonotonous way on the driving strength $K / \hbar \omega$.

\section{CONCLUSIONS}

In summary, we have investigated the conditions for Floquet engineering in optical lattices. In particular, we were interested in the existence of a frequency window where both low-frequency intraband heating and high-frequency interband heating is suppressed on a timescale $\tau$ that is large compared to the tunneling time. Considering the concrete example of a small one-dimensional system of interacting bosons in a shaken optical lattice, we presented numerical results that show that such a frequency window exists for sufficiently deep lattices. The maximum ratio of heating and tunneling time, $\tau_{\text {opt }} J_{s} / \hbar$ (which is found for an optimal intermediate driving frequency $\omega_{\mathrm{opt}}$ ), is found to increase with the lattice depth. This result, which is not obvious since also the tunneling time increases exponentially with the lattice depth, implies that we can reduce driving-induced heating by simply ramping up the lattice depth. However, we have pointed out that this strategy is limited to lattice depths for which the tunneling time is still much smaller than the timescale $\tau_{0}$ for non-driving-induced background heating. Thus, the larger the timescale for such background heating, the more we can reduce also drivinginduced heating.

We have also found that by ramping up the interaction strengths, driving-induced heating is significantly enhanced, until a saturation value is reached roughly when the ratio $U_{s} / J_{s}$ reaches values of 3 . This saturation behavior is a promising result regarding the possibility of Floquet engineering of strongly correlated states of matter such as fractional Chern insulators [49-52].

An interesting direction for future work concerns the role of disorder. It has been argued that many-body localization can protect the driven system against unwanted heating associated with deviations from the high-frequency approximation $[53,54]$. Roughly speaking, within the localization length, the system is not able to create excitations of a sufficiently large energy $\hbar \omega$. The mechanism is crucial also for the stabilization of discrete time crystals [55-61]. However, disorderinduced localization cannot be expected to prevent the system also against unwanted heating associated with deviations from the low-frequency approximation. Unwanted resonant multiphoton excitations to states above the gap can still occur. It is an interesting question, in how far the corresponding heating rates are influenced by disorder-induced localization.

\section{ACKNOWLEDGMENT}

We thank Christoph Sträter for providing the band structure data. This work was supported by the German Research Foundation DFG via the Research Unit FOR2414 (under Project No. 277974659).
[1] A. Eckardt, Colloquium: Atomic quantum gases in periodically driven optical lattices, Rev. Mod. Phys. 89, 011004 (2017).

[2] D. H. Dunlap and V. M. Kenkre, Dynamic localization of a charged particle moving under the influence of an electric field, Phys. Rev. B 34, 3625 (1986).
[3] H. Lignier, C. Sias, D. Ciampini, Y. Singh, A. Zenesini, O. Morsch, and E. Arimondo, Dynamical Control of Matter-Wave Tunneling in Periodic Potentials, Phys. Rev. Lett. 99, 220403 (2007).

[4] A. Eckardt and M. Holthaus, Ac-induced superfluidity, Europhys. Lett. 80, 50004 (2007). 
[5] C. Sias, H. Lignier, Y. P. Singh, A. Zenesini, D. Ciampini, O. Morsch, and E. Arimondo, Observation of Photon-Assisted Tunneling in Optical Lattices, Phys. Rev. Lett. 100, 040404 (2008).

[6] V. V. Ivanov, A. Alberti, M. Schioppo, G. Ferrari, M. Artoni, M. L. Chiofalo, and G. M. Tino, Coherent Delocalization of Atomic Wave Packets in Driven Lattice Potentials, Phys. Rev. Lett. 100, 043602 (2008)

[7] A. Alberti, V. V. Ivanov, G. M. Tino, and G. Ferrari, Engineering the quantum transport of atomic wave functions over macroscopic distances, Nat. Phys. 5, 547 (2009).

[8] E. Haller, R. Hart, M. J. Mark, J. G. Danzl,, L. Reichsöllner, and H.-C. Nägerl, Inducing Transport in a Dissipation-Free Lattice with Super Bloch Oscillations, Phys. Rev. Lett. 104, 200403 (2010).

[9] A. Eckardt, C. Weiss, and M. Holthaus, Superfluid-Insulator Transition in a Periodically Driven Optical Lattice, Phys. Rev. Lett. 95, 260404 (2005).

[10] A. Zenesini, H. Lignier, D. Ciampini, O. Morsch, and E. Arimondo, Coherent Control of Dressed Matter Waves, Phys. Rev. Lett. 102, 100403 (2009).

[11] A. Eckardt, P. Hauke, P. Soltan-Panahi, C. Becker, K. Sengstock, and M. Lewenstein, Frustrated quantum antiferromagnetism with ultracold bosons in a triangular lattice, Europhys. Lett. 89, 10010 (2010).

[12] J. Struck, C. Ölschläger, R. Le Targat, P. Soltan-Panahi, A. Eckardt, M. Lewenstein, P. Windpassinger, and K. Sengstock, Quantum simulation of frustrated classical magnetism in triangular optical lattices, Science 333, 996 (2011).

[13] A. R. Kolovsky, Creating artificial magnetic fields for cold atoms by photon-assisted tunneling, Europhys. Lett. 93, 20003 (2011).

[14] A. Bermudez, T. Schätz, and D. Porras, Synthetic Gauge Fields for Vibrational Excitations of Trapped Ions, Phys. Rev. Lett. 107, 150501 (2011)

[15] M. Aidelsburger, M. Atala, S. Nascimbène, S. Trotzky, Y.A. Chen, and I. Bloch, Experimental Realization of Strong Effective Magnetic Fields in an Optical Lattice, Phys. Rev. Lett. 107, 255301 (2011).

[16] P. Hauke, O. Tieleman, A. Celi, C. Ölschläger, J. Simonet, J. Struck, M. Weinberg, P. Windpassinger, K. Sengstock, M. Lewenstein, and A. Eckardt, Non-Abelian Gauge Fields and Topological Insulators in Shaken Optical Lattices, Phys. Rev. Lett. 109, 145301 (2012).

[17] J. Struck, C. Ölschläger, M. Weinberg, P. Hauke, J. Simonet, A. Eckardt, M. Lewenstein, K. Sengstock, and P. Windpassinger, Tunable Gauge Potential for Neutral and Spinless Particles in Driven Optical Lattices, Phys. Rev. Lett. 108, 225304 (2012).

[18] J. Struck, M. Weinberg, C. Ölschläger, P. Windpassinger, J. Simonet, K. Sengstock, R. Höppner, P. Hauke, A. Eckardt, M. Lewenstein, and L. Mathey, Engineering Ising- $x y$ spin models in a triangular lattice via tunable artificial gauge fields, Nat. Phys. 9, 738 (2013).

[19] M. Aidelsburger, M. Atala, M. Lohse, J. T. Barreiro, B. Paredes, and I. Bloch, Realization of the Hofstadter Hamiltonian with Ultracold Atoms in Optical Lattices, Phys. Rev. Lett. 111, 185301 (2013).

[20] H. Miyake, G. A. Siviloglou, C. J. Kennedy, W. C. Burton, and W. Ketterle, Realizing the Harper Hamiltonian with
Laser-Assisted Tunneling in Optical Lattices, Phys. Rev. Lett. 111, 185302 (2013).

[21] M. Atala, M. Aidelsburger, M. Lohse, J. T. Barreiro, B. Paredes, and I. Bloch, Observation of chiral currents with ultracold atoms in bosonic ladders, Nat. Phys. 10, 588 (2014).

[22] C. J. Kennedy, W. C. Burton, W. C. Chung, and W. Ketterle, Observation of Bose-Einstein condensation in a strong synthetic magnetic field, Nat. Phys. 11, 859 (2015).

[23] T. Oka and H. Aoki, Photovoltaic Hall effect in graphene, Phys. Rev. B 79, 081406(R) (2009).

[24] G. Jotzu, M. Messer, T. U. R. Desbuquois, M. Lebrat, D. Greif, and T. Esslinger, Experimental realization of the topological haldane model with ultracold fermions, Nature (London) $\mathbf{5 1 5}$ 237 (2014).

[25] M. Aidelsburger, M. Lohse, C. Schweizer, M. Atala, J. T. Barreiro, S. Nascimbène, N. R. Cooper, I. Bloch, and N. Goldman, Measuring the Chern number of Hofstadter bands with ultracold bosonic atoms, Nat. Phys. 11, 162 (2015).

[26] F. Görg, K. Sandholzer, J. Minguzzi, R. Desbuquois, M. Messer, and T. Esslinger, Realization of density-dependent Peierls phases to engineer quantized gauge fields coupled to ultracold matter, Nat. Phys. 15, 1161 (2019).

[27] A. Lazarides, A. Das, and R. Moessner, Equilibrium states of generic quantum systems subject to periodic driving, Phys. Rev. E 90, 012110 (2014)

[28] L. D'Alessio and M. Rigol, Long-Time Behavior of Isolated Periodically Driven Interacting Lattice Systems, Phys. Rev. X 4, 041048 (2014).

[29] S. Choudhury and E. J. Mueller, Stability of a Floquet BoseEinstein condensate in a one-dimensional optical lattice, Phys. Rev. A 90, 013621 (2014).

[30] M. Weinberg, C. Ölschläger, C. Sträter, S. Prelle, A. Eckardt, K Sengstock, and J. Simonet, Multiphoton interband excitations of quantum gases in driven optical lattices, Phys. Rev. A 92 , 043621 (2015).

[31] S. Choudhury and E. J. Mueller, Transverse collisional instabilities of a Bose-Einstein condensate in a driven one-dimensional lattice, Phys. Rev. A 91, 023624 (2015).

[32] M. Genske and A. Rosch, Floquet-Boltzmann equation for periodically driven fermi systems, Phys. Rev. A 92, 062108 (2015).

[33] T. Bilitewski and N. R. Cooper, Scattering theory for FloquetBloch states, Phys. Rev. A 91, 033601 (2015).

[34] T. Bilitewski and N. R. Cooper, Population dynamics in a Floquet realization of the Harper-Hofstadter hamiltonian, Phys. Rev. A 91, 063611 (2015).

[35] C. Sträter and A. Eckardt, Interband heating processes in a periodically driven optical lattice, Z. Naturforsch. A 71, 909 (2016).

[36] M. Reitter, J. Näger, K. Wintersperger, C. Sträter, I. Bloch, A. Eckardt, and U. Schneider, Interaction Dependent Heating and Atom Loss in a Periodically Driven Optical Lattice, Phys. Rev. Lett. 119, 200402 (2017).

[37] S. V. Rajagopal, T. Shimasaki, P. Dotti, M. Račiūnas, R. Senaratne, E. Anisimovas, A. Eckardt, and D. M. Weld, Phasonic Spectroscopy of a Quantum Gas in a Quasicrystalline Lattice, Phys. Rev. Lett. 123, 223201 (2019).

[38] K. Singh, C. J. Fujiwara, Z. A. Geiger, E. Q. Simmons, M. Lipatov, A. Cao, P. Dotti, S. V. Rajagopal, R. Senaratne, T. Shimasaki, M. Heyl, A. Eckardt, and D. M. Weld, Quantifying 
and Controlling Prethermal Nonergodicity in Interacting Floquet Matter, Phys. Rev. X 9, 041021 (2019).

[39] A. Eckardt and M. Holthaus, Avoided Level Crossing Spectroscopy with Dressed Matter Waves, Phys. Rev. Lett. 101, 245302 (2008).

[40] A. Eckardt and E. Anisimovas, High-frequency approximation for periodically driven quantum systems from a Floquet-space perspective, New J. Phys. 17, 093039 (2015).

[41] F. Casas, J. A. Oteo, and J. Ros, Floquet theory: Exponential perturbative treatment, J. Phys. A: Math. Gen. 34, 3379 (2001).

[42] A. Verdeny, A. Mielke, and F. Mintert, Accurate Effective Hamiltonians Via Unitary Flow in Floquet Space, Phys. Rev. Lett. 111, 175301 (2013).

[43] N. Goldman and J. Dalibard, Periodically Driven Quantum Systems: Effective Hamiltonians and Engineered Gauge Fields, Phys. Rev. X 4, 031027 (2014).

[44] M. Bukov, L. D'Alessio, and A. Polkovnikov, Universal highfrequency behavior of periodically driven systems: From dynamical stabilization to Floquet engineering, Adv. in Phys. 64, 139 (2015).

[45] D. Jaksch, C. Bruder, J. I. Cirac, C. W. Gardiner, and P. Zoller, Cold Bosonic Atoms in Optical Lattices, Phys. Rev. Lett. 81, 3108 (1998).

[46] W. Zwerger, Mott-Hubbard transition of cold atoms in optical lattices, J. Opt. B 5, S9 (2003).

[47] D. A. Abanin, W. De Roeck, and F. Huveneers, Exponentially Slow Heating in Periodically Driven Many-Body Systems, Phys. Rev. Lett. 115, 256803 (2015).

[48] T. Kuwahara, T. Mori, and K. Saito, Floquet-Magnus theory and generic transient dynamics in periodically driven many-body quantum systems, Ann. Phys. 367, 96 (2016).

[49] A. G. Grushin, A. Gómez-León, and T. Neupert, Floquet Fractional Chern Insulators, Phys. Rev. Lett. 112, 156801 (2014).

[50] E. Anisimovas, G. Žlabys, B. M. Anderson, G. Juzeliūnas, and A. Eckardt, Role of real-space micromotion for bosonic and fermionic Floquet fractional Chern insulators, Phys. Rev. B 91, 245135 (2015).

[51] M. Račiūnas, G. Žlabys, A. Eckardt, and E. Anisimovas, Modified interactions in a Floquet topological system on a square lattice and their impact on a bosonic fractional Chern insulator state, Phys. Rev. A 93, 043618 (2016).

[52] M. Račiūnas, F. N. Ünal, E. Anisimovas, and A. Eckardt, Creating, probing, and manipulating fractionally charged excitations of fractional Chern insulators in optical lattices, Phys. Rev. A 98, 063621 (2018).

[53] A. Lazarides, A. Das, and R. Moessner, Fate of Many-Body Localization Under Periodic Driving, Phys. Rev. Lett. 115, 030402 (2015).

[54] P. Ponte, Z. Papić, F. Huveneers, and D. A. Abanin, Many-Body Localization in Periodically Driven Systems, Phys. Rev. Lett. 114, 140401 (2015).

[55] V. Khemani, A. Lazarides, R. Moessner, and S. L. Sondhi, Phase Structure of Driven Quantum Systems, Phys. Rev. Lett. 116, 250401 (2016).

[56] D. V. Else, B. Bauer, and C. Nayak, Floquet Time Crystals, Phys. Rev. Lett. 117, 090402 (2016).

[57] S. Choi, J. Choi, R. Landig, G. Kucsko, H. Zhou, J. Isoya, F. Jelezko, S. Onoda, H. Sumiya, V. Khemani, C. von Keyserlingk, N. Y. Yao, E. Demler, and M. D. Lukin, Observation of discrete time-crystalline order in a disordered dipolar many-body system, Nature (London) 543, 221 (2017).

[58] J. Zhang, P. W. Hess, A. Kyprianidis, P. Becker, A. Lee, J. Smith, G. Pagano, I.-D. Potirniche, A. C. Potter, A. Vishwanath, N. Y. Yao, and C. Monroe, Observation of a discrete time crystal, Nature (London) 543, 217 (2017).

[59] K. Sacha and J. Zakrzewski, Time crystals: A review, Rep. Prog. Phys. 81, 016401 (2017).

[60] V. Khemani, R. Moessner, and S. L. Sondhi, A brief history of time crystals, arXiv:1910.10745.

[61] D. V. Else, C. Monroe, C. Nayak, and N. Y. Yao, Discrete time crystals, arXiv:1905.13232. 